\title{
Development of multifunction micro-pulse lidar at 1.5 micrometer Mingiia Shangguan ${ }^{1}$, Haiyun Xia ${ }^{1, *}$, Xiankang Dou ${ }^{1,2}$, Jiawei Qiu ${ }^{1}$, Chao Yu ${ }^{1}$ \\ ${ }^{l}$ School of Earth and Space Science, University of Science and Technology of China, Hefei, 230026, China \\ ${ }_{2}^{2}$ School of Electronic Information, Wuhan University, Wuhan, 430072, China \\ *Email:hsia@ustc.edu.cn
}

\begin{abstract}
Taking advantage of the $1.5 \mu \mathrm{m}$ lidar, a series of $1.5 \mu \mathrm{m}$ micro-pulse lidars have been developed at the University of Science and Technology of China, in Hefei, China. According to the different characteristics of three kinds of single-photon detectors at $1.5 \mu \mathrm{m}$, namely superconducting nanowire single-photon detector, up-conversion SPDs and InGaAs/InP single-photon avalanche diodes, different kinds of lidar systems have been constructed to realize the detection of atmospheric visibility, cloud, depolarization, wind field at the atmospheric boundary layer.
\end{abstract}

\section{INTRODUCTION}

Aerosol lidars play an important role in many fields, such as atmospheric environment management, air chemistry, hydrological cycle, radiation budget and public health. Compared with ultraviolet (UV) and visible systems, $1.5 \mu \mathrm{m}$ lidars offer several advantages, including higher maximum permissible exposure to human eyes, lower atmospheric attenuation, weaker sky radiance and minor disturbance from Rayleigh scattering.

In order to guarantee a detection range of about 10 $\mathrm{km}$, the pulse energy of the aerosol lidar should be higher than $100 \mathrm{~mJ}$ during clear weather conditions [1]. However, the peak power of such a laser pulse is far beyond the stimulated Brillouin scattering (SBS) threshold of any erbium-doped fiber amplifier (EDFA). Thus, complex and expensive solid-state techniques including stimulated Raman scattering (SRS), optical parametric oscillator, and resonator with Cr4_: YAG crystal are developed to generate highpower lasers at $1.5 \mu \mathrm{m}$ for aerosol lidars [2]. In principle, the SNR of lidar is dominated by the product of laser power and the telescope area. Although the detector used in a lidar finally decides the quality of the raw data, its contribution is often neglected. Because the quantum efficiency is usually treated as a constant, the cathode material of a detector is selected according to its sensitivity versus the working wavelength. For example, InGaAs avalanche photodiode (APD) is used for $1.5-\mu \mathrm{m}$ detection commonly.

Generally, traditional micro-pulse lidar is thought quite unsuitable for long-range aerosol detection with high temporal/spatial resolution [1]. In our work, by optimizing different single-photon detectors at $1.5 \mu \mathrm{m}$, different micro-pulse lidars incorporating a fiber laser and a small optical antenna are proposed and demonstrated for longrange atmospheric parameters detection during day and night.

\section{METHODOLOGY}

Using single-photon detectors (SPDs) at $1.5 \mu \mathrm{m}$ can greatly improve the performance of aerosol lidars. Currently, the main techniques for singlephoton detection at the telecom wavelength [2] include superconducting nanowire single-photon detectors (SNSPDs), up-conversion SPDs and InGaAs/InP single-photon avalanche diodes, as listed in Table I.

SNSPDs exhibit the excellent performance of high photon detection efficiency (PDE), low dark count rate (DCR) and low timing jitter [3]. Thus, by using the SNSPD to detect the weak backscattering with a high signal-to-noise ratio, the performance of the lidar systems can be enhanced.

The up-conversion SPDs exhibit moderate performance. It upconverts photons at communication band to visible photons when quasi-phase matching (QPM) is achieved in a periodically poled lithium niobate waveguide. Then single photons at $1.5 \mu \mathrm{m}$ can be counted by using a Si: APD with high efficiency, low noise, and less afterpulse.

Compared with the above detectors, InGaAs/InP SPADs have relatively poor performance. However, such detectors are widely used in practical applications due to their advantages of small size, multi-mode fiber (MMF) coupling, 
being polarization-independent, and low cost [4]. MMF coupling highly enhances the collection efficiency of backscattering signals distorted by atmospheric turbulence compared with SMF coupling, which, therefore, significantly reduces the volume and weight of telescopes.

Table 1 Comparison of different free-running SPDs

\begin{tabular}{cccc}
\hline & SNSPD $^{[3]}$ & UCSPD $^{[2]}$ & InGaAs $^{[4]}$ \\
\hline PDE & $93 \%$ & $20 \%$ & $10 \%$ \\
\hline DCR & $1 \mathrm{cps}$ & $60 \mathrm{cps}$ & $950 \mathrm{cps}$ \\
\hline Afterpulse & $0.0 \%$ & $0.9 \%$ & $18.0 \%$ \\
\hline Memperature & $1 \mathrm{~K}$ & $300 \mathrm{~K}$ & $223 \mathrm{~K}$ \\
\hline Fiber mode & $\mathrm{SMF} / \mathrm{MMF}$ & $\mathrm{SMF}$ & $\mathrm{SMF} / \mathrm{MMF}$ \\
\hline $\begin{array}{c}\text { Spectral } \\
\text { response }\end{array}$ & $\mathrm{Wide}$ & $\mathrm{Narrow}$ & $\mathrm{Wide}$ \\
\hline $\begin{array}{c}\text { Polarization } \\
\text { dependence }\end{array}$ & Yes/NO & Yes & NO \\
\hline 3. RESULTS & & & $1.6 \mathrm{Mcps}$ \\
\hline
\end{tabular}

\section{RESULTS}

\subsection{Visibility}
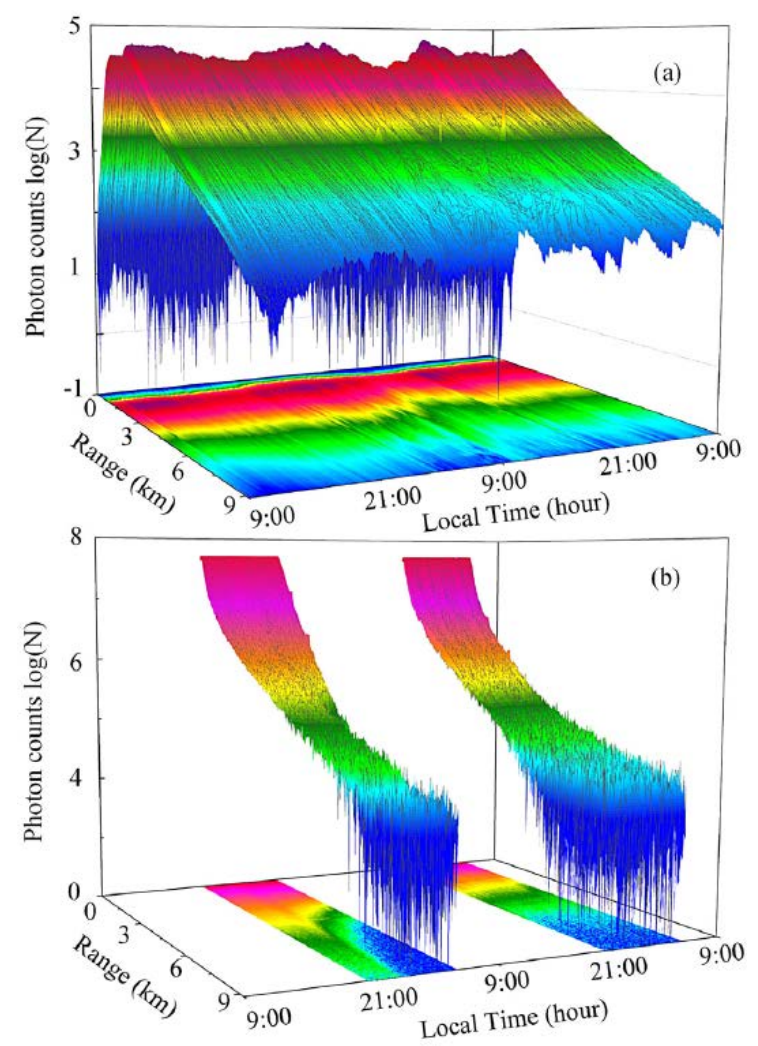

Fig. 1. Raw data of $1.5 \mu \mathrm{m}$ lidar (a) and $532 \mathrm{~nm}$ lidar (b).

An adaptive inversion algorithm for $1.5 \mu \mathrm{m}$ visibility lidar is proposed by using the particle size distribution of atmospheric aerosol [5]. The visibility with a temporal resolution of $5 \mathrm{~min}$ is detected from 9:00 on April 21 to 9:00 on April 23, 2017, in Hefei $\left(31.83{ }^{\circ} \mathrm{N}, 117.25^{\circ} \mathrm{E}\right)$. As shown in Fig. 1, $1.5 \mu \mathrm{m}$ visibility lidar can work during day and night with lower noise than 532 $\mathrm{nm}$ lidar. In the two-day experiment, the extinction coefficient at $1.5 \mu \mathrm{m}$ is always less than the extinction coefficient at $532 \mathrm{~nm}$, as shown in Fig. 2. As we can see from Fig. 3, the average visibility error between the new method and a visibility sensor (Vaisala, PWD52) is $5.2 \%$ with the R-square value of 0.96 , while the relative error between another reference visibility lidar at 532 $\mathrm{nm}$ and the visibility sensor is $6.7 \%$ with the Rsquare value of 0.91 .

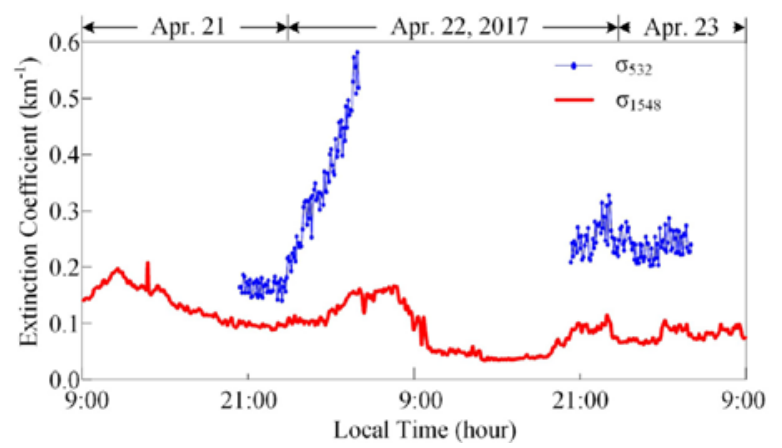

Fig. 2. The extinction coefficients of $1.55 \mu \mathrm{m}$ lidar and 532 nm lidar.

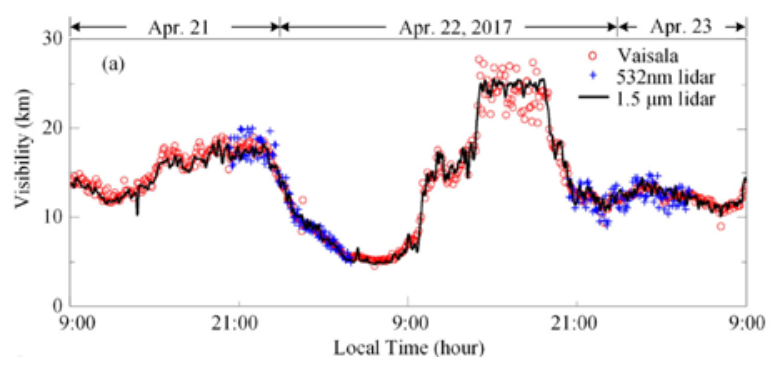

Fig. 3. Visibilities derived from different methods

\subsection{Cloud}

Since $1.5 \mu \mathrm{m}$ laser has advantages of low atmospheric attenuation and low solar background, it's an ideal wavelength for cloud detection. To collect a weak cloud signal within $15 \mathrm{~km}$, a multimode fiber (MMF) coupling InGaAs/InP singlephoton detector is developed [6]. MMF matches larger receiving telescope than single-mode fiber (SMF) due to its large diameter and high numerical aperture. Using the MMF coupling receiver can enhance the collection efficiency by about 5 times, as shown in Fig.4. A specific 
algorithm is also developed to obtain accurate cloud signals by correcting InGaAs/InP SPD noises and amplified spontaneous emission (ASE) noises of EDFA. Finally, we achieve the multilayer cloud base height and thickness detection with $15 \mathrm{~m}$ range resolution and $1 \mathrm{~s}$ time resolution. The range corrected signal of a continuous observation is shown in Fig.5.

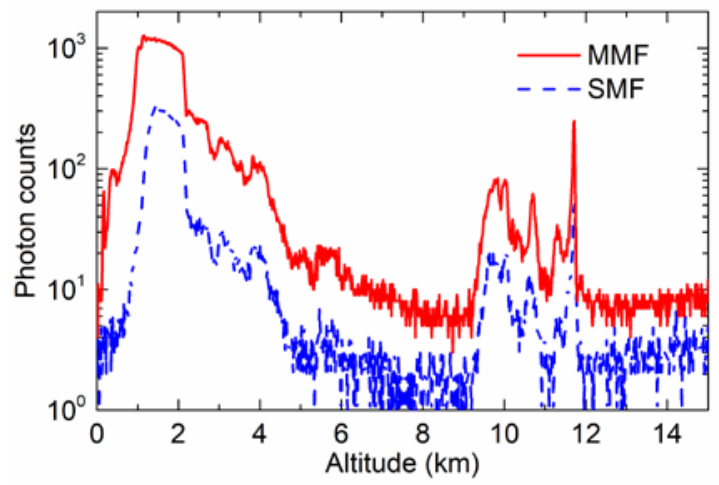

Fig. 4. Original photon counts for a vertical cloud detection observation using SMF and MMF receivers with a measurement time of $1 \mathrm{~s}$.

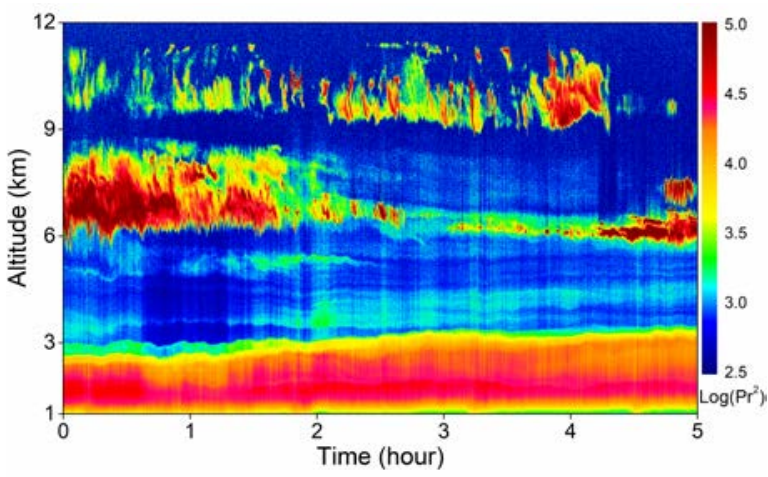

Fig. 5. Range corrected signal (log Pr2) of multi-layer clouds using the MMF receiver, observed from 00:00 to $05: 00$ on 24 March 2018.

\subsection{Polarization}

The polarization lidar has shown the capability of identifying different types of aerosols and clouds by analyzing the linear depolarization ratio (LDR). Taking advantage of the lowest attenuation $(<1$ $\mathrm{dB} / \mathrm{km}$ ) in the fiber and the maturity of optical devices at optical communication band, an allfiber eye-safe polarization lidar incorporating a time-division multiplexing (TDM) technique is developed [7]. Only a single piece of the detector is used to detect the backscatter signals at two orthogonal states in an alternative sequence. Thus, regular calibration of the two detectors in traditional polarization lidars is avoided. The signal-to-noise ratio of the lidar is guaranteed by using an SNSPD, providing high detection efficiency and low dark count noise. The LDR of the urban aerosol is observed horizontally over 48 $\mathrm{h}$ in Hefei as shown in Fig. 6.

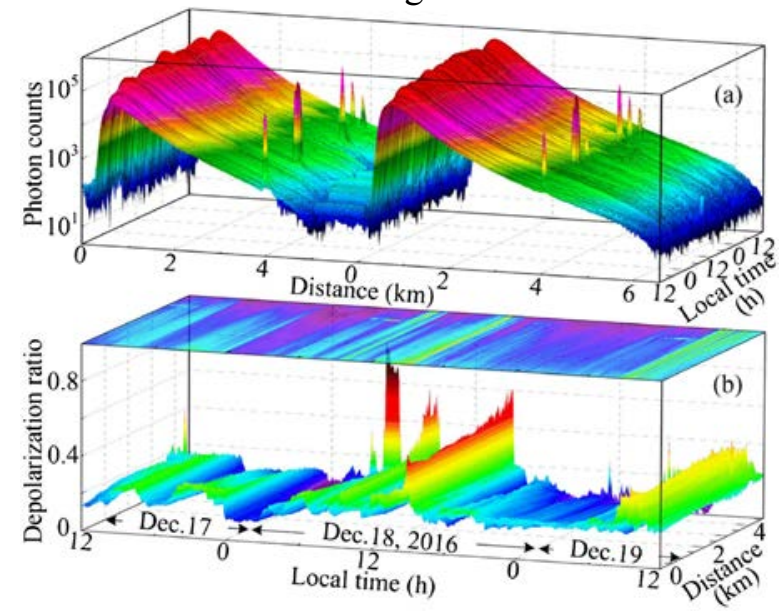

Fig. 6. (a) Raw signal of the polarization lidar over 48 $\mathrm{h}$ and (b) $48 \mathrm{~h}$ continuous measurement results of the LDR.

\subsection{Wind filed}

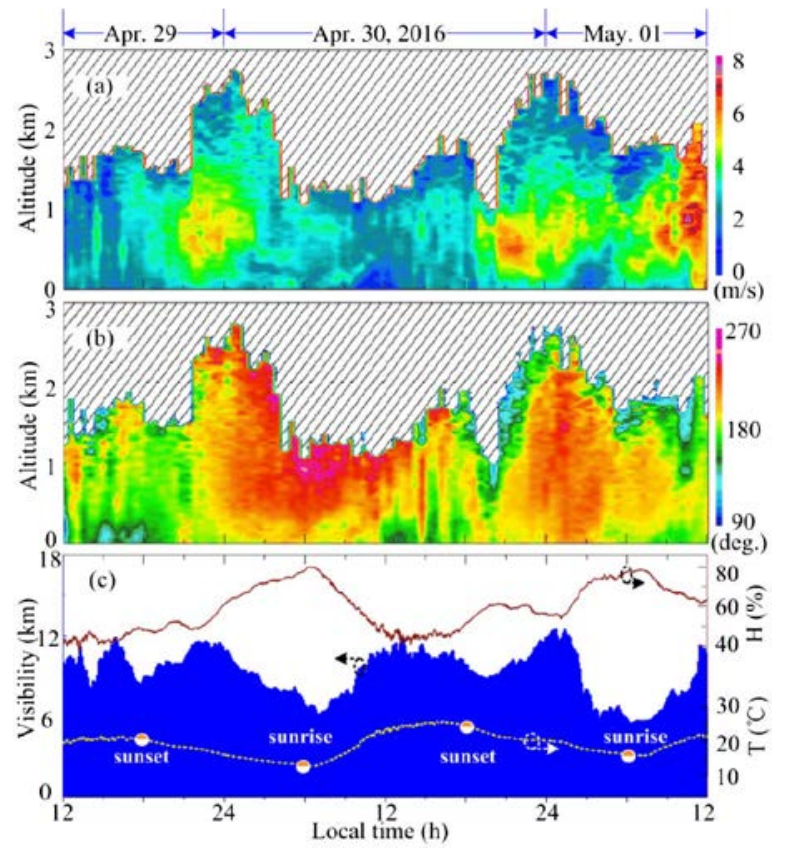

Fig. 7. Forty-eight-hour observation of atmospheric wind and visibility. (a) Wind speed, (b) direction, and (c) visibility, temperature, and humidity.

Wind field measurement is not only essential for flight safety and airport capacity, but also crucial importance for understanding the transport and dispersal processes of atmospheric pollutants [8]. High accuracy and real-time wind measurement 
will also address some vital needs such as Air Force operations, hurricane tracking, epidemic prevention and potential chemical biological release trajectory prediction.

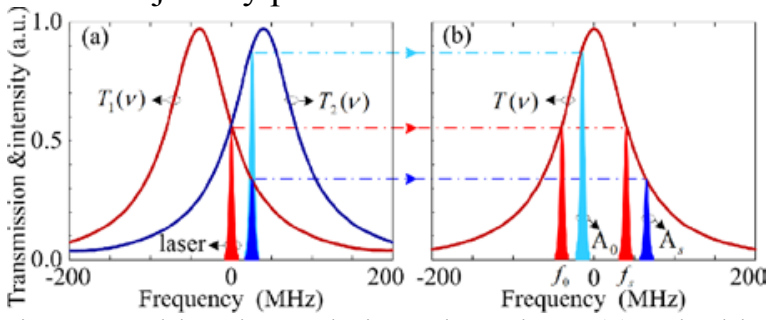

Fig. 8. Double-edge technique that adopts (a) a doublechannel FPI and (b) dual-frequency laser pulses.

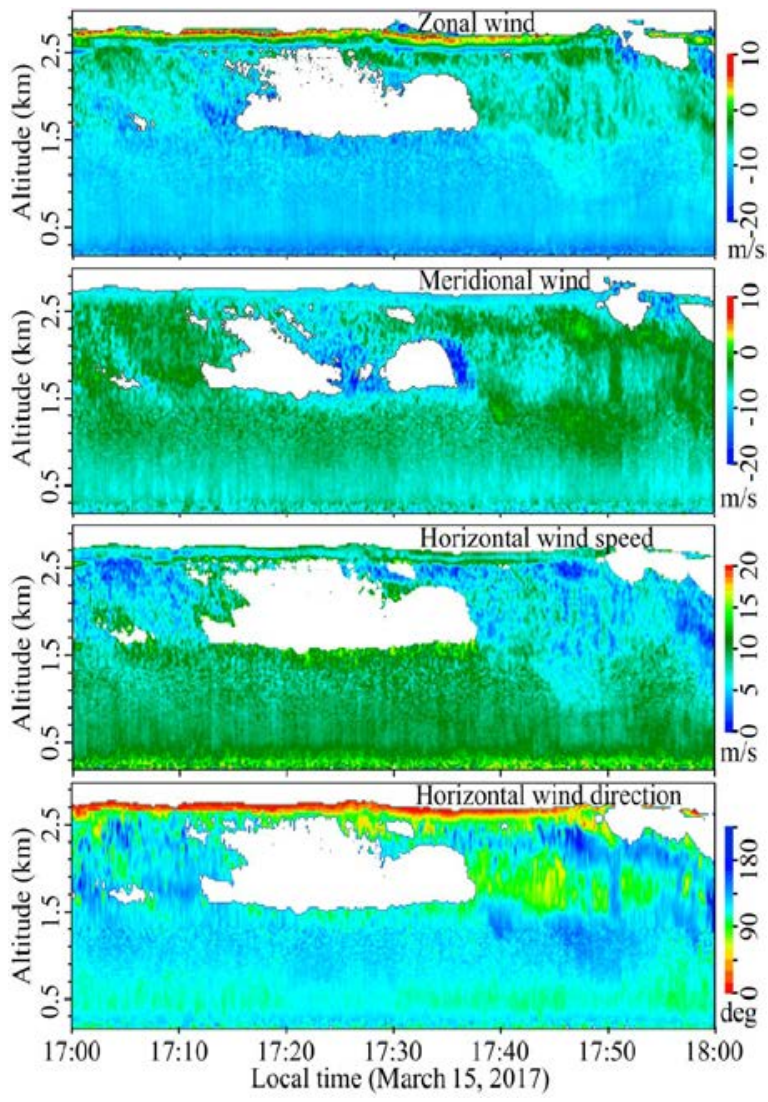

Fig. 9. One-hour observation results

By using an up-conversion single-photon detector, a versatile direct detection Doppler lidar is developed [9]. An all-fiber and polarization maintaining architecture is realized to guarantee the high optical coupling efficiency. Using integrated-optic components, the conservation of etendue of the optical receiver is achieved by manufacturing a fiber-coupled periodically poled lithium niobate waveguide and an all-fiber FabryPerot interferometer (FPI). The double-edge technique is implemented by using a convert single-channel FPI. The backscatter photons at $1548.1 \mathrm{~nm}$ are converted into $863 \mathrm{~nm}$ via mixing with a pump laser at $1950 \mathrm{~nm}$. In experiments, atmospheric wind and visibility over $48 \mathrm{~h}$ are detected in the boundary layer, as shown in Fig. 7. In order to enhance the spatial resolution, a dualfrequency direct detection Doppler lidar is developed using an SNSPD at $1.5 \mu \mathrm{m}$ [10]. The so-called double-edge technique is implemented by using dual-frequency laser pulses, rather than using a double-channel FPI, as shown in Fig. 8. Such a modification enhances the frequency stability at the system level. Two telescopes that point westward and northward at a zenith angle of $30^{\circ}$ are used to detect the line-of-sight wind components, which are used to synthesize the horizontal wind profile. Horizontal wind profiles up to an altitude of about $2.7 \mathrm{~km}$ are calculated with vertical spatial/temporal resolution of 10 $\mathrm{m} / 10 \mathrm{~s}$, as shown in Fig. 9. The dynamic evolution of wind and vertical wind shears are observed clearly.

\section{REFERENCES}

[1] S. M. Spuler and S. D. Mayor, Proc. SPIE 6681, 668102 (2007).

[2] H. Xia, G.-L. Shentu, M. Shangguan, X.-X. Xia, X. Jia, C.Wang, J. Zhang, J. S. Pelc, M. M. Fejer, Q.

Zhang, X. Dou, and J.-W. Pan, Opt. Lett. 40, 15791582 (2015).

[3] F. Marsili, V. B. Verma, J. A. Stern, S. Harrington, A. E. Lita, T. Gerrits, I. Vayshenker, B. Baek, M. D. Shaw, R. P. Mirin, and S. W. Nam, Nat. Photonics 7, 210-214 (2013).

[4] C.Yu, M. Shangguan, H. Xia, J. Zhang, X. Dou, and J.-W. Pan, Opt. Express 25, 14611-14620 (2017).

[5] X. Shang, H. Xia, X. Dou, M. Shangguan, M. Li. and C.Wang, Opt. Commn. 418, 129-134 (2018).

[6] Chao Yu, Jiawei Qiu, Haiyun Xia, Xiankang Dou, Jun Zhang, and Jian-Wei Pan, Rev. Sci. Instrum. 89, 103106 (2018).

[7] J. Qiu, H. Xia, M. Shangguan, X. Dou, M. Li, C. Wang, X. Shang, S. Lin, and J. Liu, Opt. Lett. 42, 4454-4457 (2017).

[8] M. Shangguan, H. Xia, C. Wang, J. Qiu, G.-L. Shentu, Q. Zhang, X. Dou, and J.-W. Pan, Opt. Express 24, 19322-19336 (2016).

[9] H. Xia, M. Shangguan, C. Wang, G.-L. Shentu, J. Qiu, Q. Zhang, X. Dou, and J.-W. Pan, Opt. Lett. 41, 5218-5221 (2016).

[10] M. Shangguan, H. Xia, C. Wang, J. Qiu, S. Lin, X. Dou, Q. Zhang, and J.-W. Pan, Opt. Lett. 42, 35413544 (2017). 